\title{
APORTE AL CONOCIMIENTO DE LOS AGARICALES s.l. DE CHILE
}

\section{Contribution to the knowledge of the chilean Agaricales s.l.}

\author{
E. Valenzuela*, G. Moreno**, S. Garnica1,* \\ M. Heykoop ${ }^{* *}$, M. Polette1.* \\ * Instituto de Microbiología.Facultad de Ciencias.Universidad Austral de Chile. \\ Casilla 567. Valdivia (Chile).**Departamento de Biologia Vegetal (Botánica). \\ Facultad de Ciencias,Universidad de Alcalá de Henares.28871 \\ Alcalá de Henares (Madṛid- España).
}

Palabras Clave: Agaricales s. l., taxonomia, ecología, Chile.

Key words: Agaricales s. l., taxonomy, ecology, Chile.

\section{RESUMEN}

Se citan por primera vez para Chile, 4 taxas Agaricales sensu lato, estos son: Collybia driophila (Bull.: Fr.) Kumm., Lactarius pubescens (Fr.) S. F. Gray, Lyophyllum descastes (Fr.: Fr.) Sing. y Tricholoma populinum Lange. Se aportan fotografias de los basidiocarpos y una descripción macro y microscópica de sus características principales, comentándose algunos aspectos ecológicos.

\section{INTRODUCCION}

Este trabajo representa un nuevo aporte al estudio de los Agaricales sensu lato, que fructifican en los bosques nativos y en las repoblaciones realizadas con especies alóctonas, tales como Betula, Populus y Pinus radiata en la provincia de Valdivia (Valdivia, Osorno y Llanquihue) en Chile. Otros aportes que hemos realizado en la micología chilena, son los de Valenzuela et al. (1992a, b, c,), Valenzuela etal.(1994), Moreno et al. (1993, 1994), Valenzuela \& Moreno (1994) y Valenzuela \& Esteve-Raventos (1994). En ellos además de las descripciones de taxa, se entrega gran parte de la bibliografía micológica chilena existente, sobre los Agaricales s. l. y Gasterales s. $l$.

\section{MATERIALES Y METODOS}

Las preparaciones microscópicas han sido realizadas en $\mathrm{KOH} 5 \%$, rojo congo amoniacal, agua destilada y reactivo de Melzer. Las reacciones químicas sobre la cutícula de los basidiocarpos, láminas y carne se realizaron con $\mathrm{KOH} 10 \%$ y ácido sulfúrico. El material estudiado se conserva en el herbario del Departamento de Biología Vegetal (Botánica), de

\section{SUMMARY}

Collybia driophila (Bull.: Fr.) Kumm., Lactarius pubescens (Fr.) S. F. Gray, Lyophyllum descastes (Fr:: Fr.) Sing. and Tricholoma populinum Lange ar reported for the first time for Chile. A photographies, macro and microscopic descriptions are given and ecological aspects ar commented.

la Universidad de Alcalá de Henares (AH) y un duplicado en el herbario particular (E. Valenzuela), Universidad Austral de Chile.

\section{RESULTADOS Y DISCUSIÓN}

Collybia driophila (Bull.:Fr.) Kumm.

Material Estudiado: En restos de troncos enterrados en el suelo. Jardín Botánico de la Universidad Austral de Chile, Valdivia 31-III-1993, leg. S. Garnica, Ah 16888.

Pileo de $1-5 \mathrm{~cm}$ de diám, convexo a plano-convexo 0 plano, higrófano, glabro, de color marrón-rojizo, más pálido o crémeo-amarillento a anaranjado-amarillento hacia el margen. Margen incurvado a plano-decurvado, entero, estriado, de color crémeo-amarillento. Láminas sinuadas o adnatas, estrechas, blancas, cremas en la madurez. Estípitede 3-7 x0.3$0.5 \mathrm{~cm}$, cilíndrico, liso, finamente estriado en la madurez, glabro, de color blanco o concoloro con la lámina hacia el ápice, hacia abajo del color del píleo, pero pálido, la base con cordones miceliales blanquecinos. Carne escasa, de colsr crémeo, con el ácido sulfúrico se torna violeta, con $\mathrm{KOH} 10 \%$ negativa. Olor y sabor fúngico. Esporada blanca. Esporas de $5-6(7) \times 2.8-3.5 \mu \mathrm{m}$, dacrimoides, lisas, hialinas, no amiloides. Basidios de 15-20 x 5-7 $\mu$ m, claviformes, tetraspóricos, hialinos. Queilocistidios de 15-40 x 3-5 $\mu \mathrm{m}$ filiformes, diverticulados, 
hialinos. Pleurocisticidios ausentes. Pileipillis filamentosa formada por hifas de 5-10 $\mu \mathrm{m}$ diám., ramificadas, no diverticuladas, fibuladas y pigmentadas con incrustaciones marrones.

Observaciones: Especie que fructifica en forma gregaria o cespitosa en bosque de coníferas, caducifolios, en bordes de caminio y parques, sobre el humus, entre restos leñosos depositados en el suelo o enterrados.

De acuerdo a Halling (1983), Collybia driophila es la más común y ampliamente distribuida especie de Collybia y generalmente se compara con $C$. butyracea, esto lleva a confusión ya que ambas no están presentes durante la misma estación del año, además $C$. butyracea presenta esporada con tintes rosados, las esporas son dextrinoides, cianófilas, las hifas de la pileipellis presentan una ordenación diferente y la base del estípite no presenta cordones miceliales.

\section{Lactarius pubescens (Fr.) S.F.Gray (Fig. 1)}

Material estudiado: En cesped de jardín, alrededor de Betula pendula. Jardines de la Universidad Austral de Chile, Valdivia, 22-IV-1994, leg. E. Valenzuela y S. Garnica, AH 16889. En jardines con Betula pendula. Calle Anibal Pinto, Valdivia, 26-V-1994, leg. E. Valenzuela y M. Heykoop, AH 16890.

Píleo de 4.5 - $7.5 \mathrm{~cm}$ de diám., convexo deprimido o umbilicado, seco, el centro de color ocre oscuro y hacia el margen ocré-crémeo sin zonación. Margen encurvado cubiertos de pelos de apariencia lanosa de color blanquecino a crémeo. Láminas decurrentes, estrechas, blancas con reflejos rosados. Arista entera y concolora a la lámina. Estípite de $3-5 \times 1-1.5 \mathrm{~cm}$, cilíndrico o con la base más ensanchada, hueco, escrobiculado, de color blanco-rosado, hacia las láminas rosado carne. Carne espesa, de color blanco -crémeo con $\mathrm{KOH}$ 10\% vira debílmente al amarillo. Látex de color blanquecino inmutable y de sabor picante. Esporas de (6) 7-8.5 (9) x5-6 (6.5) $\mu \mathrm{m}$, ovoides, hialinas, verrucosas, reticuladas a subretículadas, amiloides. Basidios de 25-35 x5$7 \mu$ mclaviformes, hialinos. Cistidiosde $30-45 \times 6 \mu \mathrm{m}$, cilíndricos a subclaviformes. Pileipellis formada por hifas de 3-5 $\mu \mathrm{m}$ de diám, hialinas, marrones en $\mathrm{KOH} 10 \%$, sin fibulas.

Observaciones: Lactarius pubescens se caracteriza por su píleo sin zonación, látex blanquecino inmutable de sabor picante, por sus medidas esporales y por establecer micorrizas con especies del género Betula. De acuerdo a Moreno et al .(1986), en Europa $L$. pubescens establece micorrizas con Betula pubescens, este mismo autor señala que especies próximas a $L$. pubescens son L. torminosus (Schaeff.: Fr) S.F. Gray, que presenta un píleo de color rosado y zonado, margen con abundantes excrecencias lanosas, carne y látex blanquecino de sabor muy picante y está asociado a especies del género Betula. L. tesquorum Malençon y L. mairei Malençon, carecen de coloraciones rosadas en la cutícula y presentan ecológias diferentes; el primero fructifica con Cistus spp. y el segundo en bosques del género Quercus . L. deliciosus Fr. se diferencia por su cutícula glabra, látex anaranjado y fructifica en bosques de coníferas (Pinus radiata) en Chile.

Arora (1986), indica que $L$. pubescens fructifica desde el Este de Norte América a Idaho; Colorado en parques, jardínes y bosques que presentan especies del género Betula y en forma conjunta con L. torminosus.

Lyophyllum decastes (Fr.:Fr.) Sing.

Material estudliado: En suelo, alrededor de Populus nigra. Alameda de la Universidad Austral de Chile, Valdivia, 18-VIII-1993, leg. E. Valenzuelay S. Garnica, AH 16891.

Fructificaciones que crecen en forma fascículada. Píleo de 3.5-8 cm de diám., convexo a planoconvexo, con un mamelón obtuso al centro, elástico. Cutícula lisa con pequeñas fibrillas radiales, brillante, húmeda, de color marrón a marrón -ocráceo, más claro hacia el margen llegan-do a ser crémeo a blanquecino. Margen debílmente estriado, incurvado crémeo a blanquecino. Láminas subdecurrentes por un corto diente, apretadas, de color blanco puro, con la arista entera y concolora a la lámina. Estípite de 4.5-10 x0.5-1.5 (2) cm, cilíndrico a veces más engrosado o afinandose hacia la base, elástico, recto o curvado, glabro, fibroso, blanquecino. Olor y sabor fúngicos. Esporada blanquecina. Esporas de 5.5 - 7 × 5- $6.6 \mu \mathrm{m}$, subglobosas, lisas, hialinas. Basidios de 30-45 x 8-10 $\mu \mathrm{m}$, claviformes, tetraspóricos, carmonófilos con el carmín-acético. Cistidios no observados. Pileipillis formada por hifas cilíndricas de 4-10 $\mu \mathrm{m}$, montadas en agua presentan un pigmento de color cafesoso, fibulas presentes.

Observaciomes: Lyophylum decastes se caracteriza por su crecimiento fasciculado, su píleo de color marrón, láminas y estípite blancos. De acuerdo a Moreno et al. (1986) y Breitenbach \& Kranzlin (1991), L. decastes es una especie próxima a una serie de taxa que han sido separadas por pequeños detalles y por algunos micólogos como Moser (1983), consideradas como variedades, así por ejemplo $L$. loricatum $(\mathrm{Fr}$.) Kühner [=Tricholoma cartilagineum (Bull.: Fr.) Gillet ], de microscopia semejante a $\boldsymbol{L}$. decastes y pileo con cutícula gruesa-cartilaginosa de color marrón-castañao. Es considerado por Romagnesi (1977) como una variedad de $L$. decastes.

L. decastes presenta una amplia distribución mundial, fructifica en suelo de bosques caducifolios, coníferas, parques, bordes de caminos ricos en humus, formando gruesos fascículos alrededor de tocones, troncos y raíces enterradas de árboles. L. decastes, es considerado por Moreno et al. (1986), una buena especie comestible que por su crecimiento fascículado recompensa a la persona que lo descubre en el campo, por el gran número de carpóforos que puede recoger.

\section{Tricholoma populinum Lange. (Fig.2)}

Material estudiado: En suelo, alrededor de Populus nigra. Jardín Botánico Universidad Austral de Chile, Valdivia, 16-IV-1992, leg. S. Garnica y J.Grinbergs, AH15506.

Píleo de 6-12 (15)cm de diám., convexo a plano-convexo. Cutícula glabra, lisa, viscosa en tiempo húmedo, de color marrón claro a marrón-rojizo, más oscura al centro. Margen encurvado a decurvado, liso, concoloro a la cutícula o un 
Fig 1.- Basidiocarpos de Lactarius pubescens

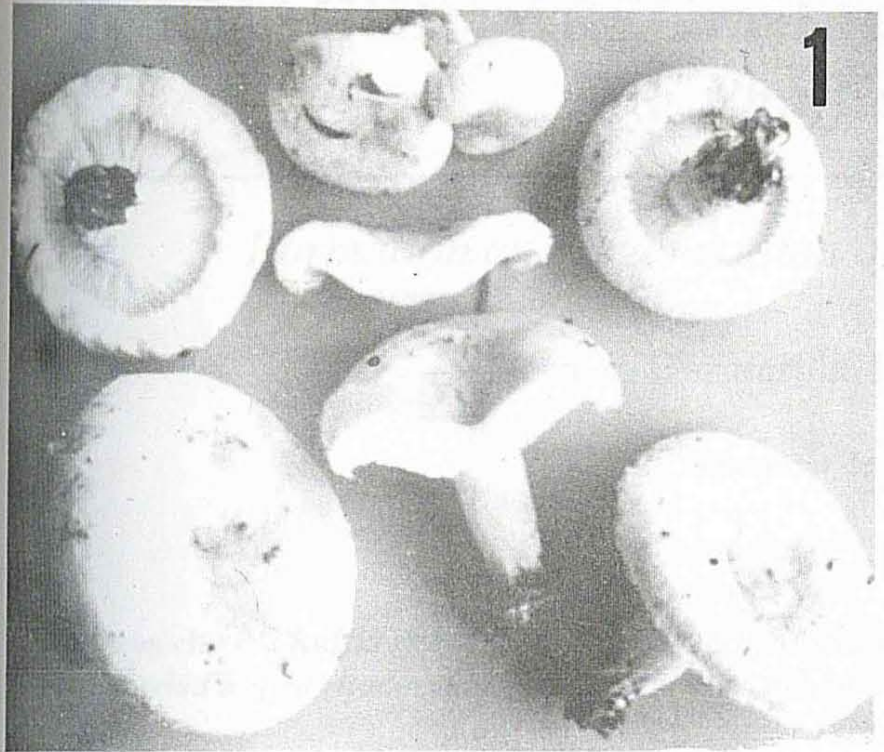

poco más pálido. Láminas adnatas, ventrudas, apretadas, de color blanco tornándose marrón-rojizo con la edad, la arista entera y concolora a la lámina o de color marrón pálido. Estípite de 5-12(15) x 1-3 cm, cilíndrico a claviforme, blanco tornándose marrón o crémeo-marrón pálido, robusto, finamente fibriloso longitudinalmente. Carne firme, blanca a rojo-marrón pálida hacia la cutícula y el margen del estípite. Olor farinoso. Sabor dulzaino pasando a farinoso. Esporada blanca. Esporas de 4-6.5 ×3-4.5 $\mu \mathrm{m}$, anchamente elipsoidales, lisas, hialinas, no amiloides. Basidios de 20-35 x 5-8 $\mu \mathrm{m}$, cilíndrico- claviformes, tetraspóricos, hialinos. Cistidios no observados. Pileipillis formada por hifas cilíndricas de 4-8 $\mu \mathrm{m}$ de diám., con pigmento de color rojo-marrón en $\mathrm{KOH} 10 \%$. Fibulas presentes.

Observaciones: Especie que se caracteriza por su píleo subviscoso en estado húmedo, de color marrón con tintes rojizos; láminas que oscurecen en la vejez; estípite sin zona anular, por su crecimiento fascículado y su ecología, fructifica en suelo, bajo Populus spp.

Breintenbach \& Krazlin (1991), señalan que la especie próxima a Tricholoma populinum es $T$. pessundatum (Fr.) Quél., difiere por su ecología, ya que fructifica en bosques de coniferas especialmente Pinus y Picea y por sus esporas más anchas.

\section{Agradecimientos.}

Queremos agradecer a la DID, proyecto S-94-01 Univ. Austral de Chile, por el financiamiento para el estudio de los Agaricales de la zona de Valdivia. A la Agencia Española de Cooperación Internacional del Ministerio de Asuntos Exteriores, Secretaria de Estado para la Cooperación Internacional y para Iberoamérica, la concesión a uno de nosotros (E. Valenzuela) de una beca para la realización del Doctorado en la Universidad de Alcalá de Henares.

\section{REFERENCIAS}

Arora, D. (1986). Mushrooms demystified a comprehensive guide to
Fig 2.- Basidiocarpos de Trichiloma populinum

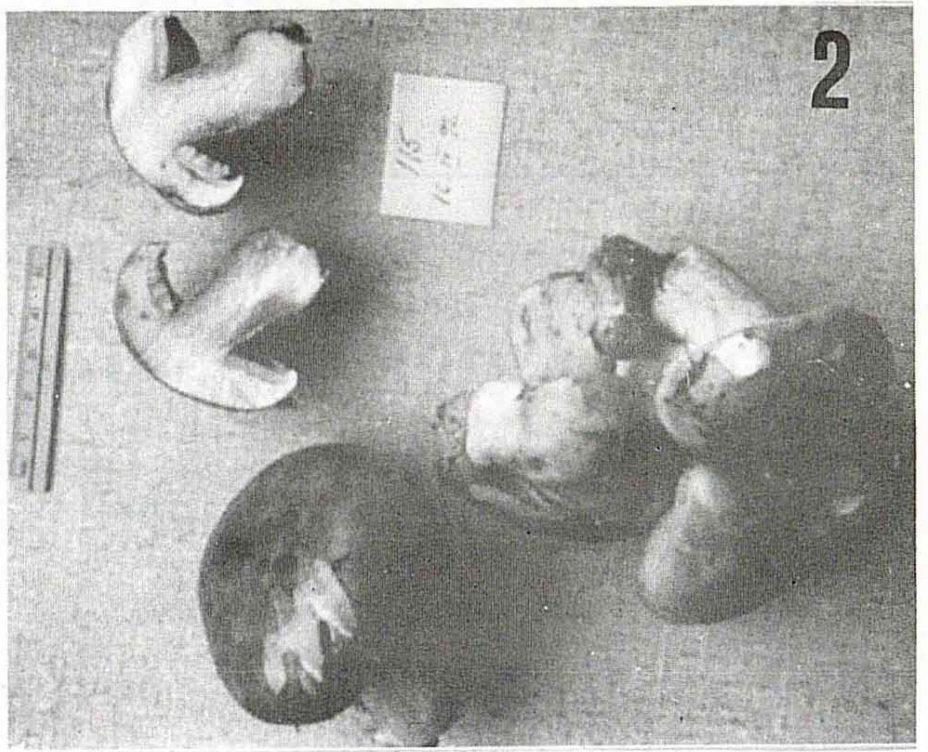

the fleshy fungi. Ed. Ten Speed Press.

Breintenbach, J. \& Kranzlin, E. (1991). Champignons de Suisse, contribution á la connaisence de la flore fongique de Suisse. Edition Mycologia Lucerne.

Hallin, R.E. (1983). The genus Collybia (Agaricales) in the northeastern United States and adjacent Canada. J. Cramer, Germany.

Moreno, G.; Manjón, J.L.; Zugaza, A. (1986).La guía de Incafo de los hongos de la Península Ibérica. Vols. I y II Incafo. Madrid.

Moreno, G.; Ochoa, C.; Valenzuela E. (1993). Bovista pila Berk \& Curtis y scleroderma bovista Fr. (Gasteromycetes) en Chile. Bol. Soc. Micol. Madrid 18: 145-150.

Moreno, G.; Valenzuela, E.; Marchetti, M. (1994). Pholiota variicystis sp. nov. dal Cile. Bol. Dell. Assoc. Micol. Bresadola 34 : $35-$ 38.

Moser, M. (1983). Kleine Kryptogamenflora, Bnd II b/ II. die $\mathrm{R}<148>$ hrlinge und blatterpilze, 5, Auflage. Stuggart.

Romagnesi, H. (1977). Champignons d' Europe. 2 Vols. Bordas. Paris.

Valenzuela, E.; Moreno, G.;Grinbergs, J. (1992a). Agaricales sensu lato de Chile. I. Bol. Soc. Micol. Madrid 17: 81-93.

Valenzuela, E.; Moreno, G. ; Grinbergs, J. (1992b). Algunas Boletaceas que fructifican en el sur de Chile. Bol. Micol. Chile. Chile 7: 1-15.

Valenzuela, E.; Moreno, G. ; Grinbergs, J. (1992c). Amanita Phalloides en bosques de Pinus radiata de la IX Región de Chile: taxonomia, toxinas, métodos de detección, intoxicación faloidiana. Bol. Micol.Chile 7: 17-21.

Valenzuela, E.; Moreno, G.; Garnica, S. \& Grinbergs, J. (1994). Agaricales sensu lato de Chile II.Bol. Micol. Madrid 19: 281-304.

Valenzuela, E. \& Moreno, G. (1994). Colbybia grinbergsii sp. nov. from Chile. Mycotaxon 60: 489-493.

Valenzuela, E. \& Esteve- Raventos. (1994). Cortinarius horakii, a new species from Chile. Mycol. Res. 98: 397-398. 